\title{
Fabrication of psoralen-loaded lipid-polymer hybrid nanoparticles and their reversal effect on drug resistance of cancer cells
}

\author{
YUELING YUAN $^{1 *}$, PETER CHIBA $^{2 *}$, TIANGE CAI $^{3 *}$, RICHARD CALLAGHAN $^{4}$, \\ LI BAI ${ }^{5}$, SUSAN P.C. COLE $^{6}$ and YU CAI ${ }^{1,7}$ \\ ${ }^{1}$ College of Pharmacy, Jinan University, Guangzhou, Guangdong 510632, P.R. China; ${ }^{2}$ Institute of Medical Chemistry, \\ Medical University of Vienna, 1090 Vienna, Austria; ${ }^{3}$ College of Life Science, Liaoning University, Shenyang, \\ Liaoning 110000, P.R. China; ${ }^{4}$ Research School of Biology, Australian National University, Canberra 2601, \\ Australia; ${ }^{5}$ Guangzhou Jiayuan Pharmaceutical Technology Co., Ltd., Guangzhou, Guangdong 510663, \\ P.R. China; ${ }^{6}$ Division of Cancer Biology and Genetics, Queen's University, Kingston, ON K7L 3N6, \\ Canada; ${ }^{7}$ Cancer Research Institute, Jinan University, Guangzhou, Guangdong 510632, P.R. China
}

Received January 20, 2018; Accepted June 13, 2018

DOI: $10.3892 /$ or.2018.6492

\begin{abstract}
In the present study, a lipid-polymer hybrid drug carrier system was developed to encapsulate psoralen (PSO), a multidrug resistance reversal agent and traditional Chinese medicine. Emphasis was focused the parameters that influence physicochemical characteristics, and then the drug release profile, stability, cytotoxicity and drug resistance reversal effect of the lipid-polymer hybrid nanoparticles (LPNs) were investigated. It was found that various formulation parameters affected NP size, drug loading (DL) and release characteristics. Hydrophilic 1,2-distearoyl-sn-glycero3-phosphoethanolamine-N-carboxy(polyethylene glycol)2000 increased the $\zeta$ potential and thus the stability of the NPs, but also enlarged their diameter. The amount of PSO influenced their DL and encapsulation efficiency, but did not show any effect on drug release kinetics. Next, the stability of the LPNs in different media and their storage characteristics were assessed. Finally, the cytotoxicity and multidrug resistance reversal effect was studied in the K562 and HepG2 cell lines.
\end{abstract}

Correspondence to: Professor Susan P.C. Cole, Division of Cancer Biology and Genetics, Queen's University, 10 Stuart Street, Kingston, ON K7L 3N6, Canada

E-mail: spc.cole@queensu.ca

Professor Yu Cai, College of Pharmacy, Jinan University, 601 Huangpu Avenue West, Tianhe, Guangzhou, Guangdong 510632, P.R. China

E-mail: caiyu8@sohu.com

*Contributed equally

Key words: lipid-polymer hybrid nanoparticles, psoralen, controlled drug release, drug delivery, multidrug resistance
The analysis of half maximal inhibitory concentration values demonstrated that combination therapy with doxorubicin (DOX) and PSO-loaded LPNs (P-LPNs) was 14- and 23-fold more effective than a single-dose DOX treatment in resistant K562 and HepG2 cells, respectively, and 2.2- and 2.1-fold more effective than a single-dose combination regimen of DOX and PSO in solution, respectively. These data indicate that the LPNs have superior properties compared with a combination therapy in solution.

\section{Introduction}

The use of nanoparticle (NP) technology in cancer chemotherapy results in a superior performance. Advantageous properties include enhanced solubility of hydrophobic drugs, the possibility of surface functionalization for targeted delivery, and controlled and sustained drug release, which allows a reduction in drug dosage and thus fewer drug side effects $(1,2)$. In addition, it has been confirmed that NP therapy could take advantage of the enhanced permeability and retention (EPR) effect in solid tumors. The EPR effect results from an abnormal lymphatic drainage system in tumors, which increases the accumulation of nanocarrierdelivered drugs, as the tumor fails to remove NPs from the interstitial space (3).

Liposomes and polymeric NPs are the two most prevalently advocated nanocarriers for anticancer drug delivery $(4,5)$. Liposomes show superior bio-compatibility, enhanced loading efficiency and a favorable pharmacokinetic profile, including prolonged circulation time. Easy surface functionalization for targeted delivery is an additional benefit. Therefore, liposomes represent an increasingly applied drug delivery vehicle (6). Although liposomes have the aforementioned advantages, burst release of drugs, instability and lack of structural integrity represent limitations of the system $(7,8)$.

Biodegradable polymeric NPs improve the solubility of hydrophobic drugs, promote stability and enable controlled 
release. Thus, this system was developed as a second predominant platform for drug delivery (9). However, NPs formed by biodegradable polymers do not possess the same favorable biocompatibility profile as liposomes, with lower cellular uptake in comparison. Thus, a delivery system combining the advantages of liposomes and polymer NPs, while avoiding disadvantages, would be considered as ideal. Recent studies have reported that lipid-polymer hybrid NPs (LPNs) with a lipid shell and a polymer core, improve stability, enhance cellular uptake and increase encapsulation efficiency (EE) $(5,10,11)$. LPNs feature a polymer core coated with lipid layers, thereby combining the superior biocompatible property of lipids with the structural integrity of polymeric NPs. Poly(D,L-lactide-coglycolide) (PLGA), one of the Food and Drug Administration (FDA)-approved biodegradable polymers, is most commonly used as the polymer core, where poorly water-soluble drugs are encapsulated (12). Adding a lipid layer onto PLGA NPs gives rise to LPNs.

The lipid component always contains natural phospholipids, including lecithin, soybean phospholipids and synthetic phospholipids, including 1,2-distearoyl-sn-glycero3-phosphoethanolamine-N-carboxy(polyethylene glycol)2000 $\left(\mathrm{DSPE} \mathrm{PEG}_{2000}\right)$. Phospholipids, which contain hydrophilic headgroups and two hydrophobic chains, form a monolayer around the hydrophobic polymercore, while the DSPE-PEG2000 embeds in the lipid monolayer to form a PEGylation stealth layer outside the lipid shell, thus promoting electrostatic and steric stability and prolonged circulation times (Fig. 1A) (13).

Psoralen (PSO), a natural coumarin compound, which is popular in traditional Chinese medicine, has antitumor, estrogen-like and multi-drug resistance modulating properties (14-16). However, its use has been limited due to poor solubility. Recently, various drug delivery systems have been developed to improve solubility and controlled release. Jambhrunkar et al (17) developed mesoporous silica nanoparticles to encapsulate griseofulvin, with low water solubility. Results revealed that a charged surface improved the solubility and drug release of griseofulvin, while hydrophobic modification on the nanoparticle led to sustained drug release. Resveratrol cross-linked chitosan nanoparticles modified with phospholipids were synthesized by Jeong et al (18). Data revealed that the chitosan nanoparticles increased the water solubility of resveratrol and displayed slow and sustained release. A methotrexate loaded lipid-polymer hybrid nanocarrier consisting of PLGA and Lipoid S100 was prepared, which showed superior controlled action compared with the free drug solution (19). In the present study, an emulsificationsolvent-evaporation (ESE) method was employed to fabricate PSO-loaded LPNs (P-LPNs) (20). Emphasis was focused on optimizing the parameters for production of LPNs and finding an optimal formulation with respect to the in vitro drug release, storage properties and cytotoxicity. The size, poly-dispersity and $\zeta$ potential of the LPNs were characterized by dynamic light scattering (DLS). Transmission electron microscopy (TEM) was used to monitor surface morphology and structure. EE, drug loading (DL) and drug-release kinetics were determined by high-performance liquid chromatography (HPLC). The resistance reversal effect of P-LPNs proved superior to free PSO, which was shown by in vitro cytotoxicity assays in K562 and HepG2 cells.

\section{Materials and methods}

Materials. PSO ( $>98 \%$ purity) was purchased from Chengdu Pufeide Biotechnology Co., Ltd. (Sichuan, Chengdu, China). DOX hydrochloride was obtained from Selleck Chemicals (Houston, TX, USA). Soybean phospholipids [injection grade; phosphatidylcholine (PC)] were produced by Shanghai Tywei Pharmaceutical Co., Ltd. (Shanghai, China). 1,2-Distearoylsn-glycero-3-phosphocholine-N-[methoxy(polyethylene glycol)-2000 (DSPE-PEG2000) was provided by Shanghai AVT Pharmaceutical Technology Co., Ltd. (Shanghai, China). Poly(d,l-lactide-co-glycolide) (PLGA; 50:50; molecular weight, 8,000-12,000) was obtained from Jinan Daigang Biomaterial Co., Ltd. (Shandong, Jinan, China). Glucose, lactose, mannitol, sucrose and D-trehalose were provided by Tianjin Damao Chemical Company (Tianjin, China). Cell Counting Kit-8 (CCK-8) was obtained from Dojindo Molecular Technologies, Inc. (Kumamoto, Japan). RPMI1640 cell culture medium, penicillin-streptomycin and trypsin-EDTA were purchased from Thermo Fisher Scientific, Inc. (Waltham, MA, USA). Fetal bovine serum (FBS) was provided by AusGenex Pty, Ltd. (Molendinar, Queensland, Australia). Dialysis membranes with a molecular weight cutoff of 8,000-14000 KDa were purchased from Sangon Biotech, Co., Ltd. (Shanghai, China). The chronic myeloid leukemia K562 and hepatoblastoma HepG2 cell lines were provided by Nanjing KeyGen Biotech Co., Ltd. (Nanjing, Jiangsu, China). Ethanol, acetonitrile and all other chemicals were HPLC grade.

Preparation of the NPs. The ESE technique was used to prepare the P-LPNs. Briefly, PLGA (1.9 to $75 \mathrm{mg} / \mathrm{ml})$ and PSO $(0.75-2.25 \mathrm{mg} / \mathrm{ml})$ were dissolved in acetonitrile at room temperature, which served as the organic phase. Phospholipids and DSPE-PEG2000 (4:1 mass ratio) were first dissolved in ethanol at $100 \%$ of the PLGA polymer weight, then water was added to obtain a $4 \%$ ethanol solution, which acted as the aqueous phase. The mixture was heated to $70^{\circ} \mathrm{C}$ and the organic phase was added dropwise into the preheated aqueous phase, with an oil-water ratio of 1:10 (v/v). The solution was stirred for $90 \mathrm{~min}$ to evaporate the organic solvent and allow self-assembly. The NP emulsion was filtered through a $0.22-\mu \mathrm{m}$ membrane. P-LPNs were used immediately, stored at $4^{\circ} \mathrm{C}$ or lyophilized for later use.

Characterization of NPs. Particle size, polydispersity and $\zeta$ potential were measured by DLS using a Zetasizer Nano ZS90 (Malvern Panalytical, Malvern, England). The dispersion of NPs was diluted with ultrapure water and subsequently analyzed.

EE and DL capacity. The EE and DL capacity were measured using an ultrafiltration method. The NP emulsion was centrifuged at $11,000 \mathrm{xg}$ for $30 \mathrm{~min}$ at $4^{\circ} \mathrm{C}$ to collect free PSO, which was then determined by HPLC (Agilent 1260; Agilent Technologies, Inc., Santa Clara, CA, USA) at an absorbance wavelength of $245 \mathrm{~nm}$. Another aliquot of the P-LPN solution was extracted by organic solvent to obtain total PSO in the NP emulsion. The HPLC was equipped with a reverse-phase column (Agilent Technologies, Inc.; 150x4.6 mm; $5 \mu \mathrm{m}$ ). 
The mobile phase was a mixture of acetonitrile and water $(55: 45 \mathrm{v} / \mathrm{v})$ and the flow rate was $1.0 \mathrm{ml} / \mathrm{min}$. The DL capacity and $\mathrm{EE}$ were calculated as follows $(5,8): \mathrm{EE} \%=$ (total drug in $\mathrm{NP}$ - free drug)/total drug in NP $\times 100$. DL\% = encapsulated drug / total drug in NP x 100.

TEM. The morphology of the NPs was determined by TEM (TECNAI 10; Philips Healthcare, Amsterdam, The Netherlands) at an accelerating voltage of $200 \mathrm{kV}$. One drop of the NP dispersion was deposited onto the carboncoated copper grid, followed by air-drying at room temperature overnight prior to imaging. Samples on the copper grid were scanned at x37,000 magnification.

In vitro PSO release. The dialysis-bag method was used to investigate PSO release. Phosphate-buffered saline (PBS; $0.1 \mathrm{M}, \mathrm{pH} 7.4$ ) containing $0.1 \%$ (w/v) Tween-80, was used as the release medium to improve the solubility of PSO. Dialysis bags containing $2 \mathrm{ml} \mathrm{NP}$ solution were placed into a beaker filled with $200 \mathrm{ml}$ PBS. Subsequently the beaker was incubated at $37^{\circ} \mathrm{C}$ in a water bath under continuous stirring at $100 \mathrm{rpm}$. At the indicated times, a $1 \mathrm{ml}$ aliquot of the release medium was removed for determining PSO content by HPLC analysis. The release medium was exchanged periodically during the dialysis process.

Stability studies. In order to investigate the in vitro stability of NPs in solution, an aliquot of NP solution was filtered through a $0.22-\mu \mathrm{m}$ membrane and incubated in five volumes of PBS, RPMI-1640 medium, RPMI-1640 medium with 10\% FBS, $10 \%$ (v/v) human plasma in PBS and $100 \%$ human plasma at $37^{\circ} \mathrm{C}$ for $120 \mathrm{~h}$. Particle size and polydispersity were measured by dynamic light scattering at 24 -h intervals.

Optimizing the formulation of lyoprotectant. Freeze-dried drugs can be stored for a prolonged period of time and easily be re-dissolved to restore activity. Thus, freeze-drying technology is widely used in the preparation of NPs. In order to develop appropriate storage conditions, five different lyoprotectants, including glucose, lactose, mannitol, sucrose and D-trehalose, were tested. NPs were frozen and kept at $-80^{\circ} \mathrm{C}$ overnight, then lyophilized at $-80^{\circ} \mathrm{C}$ for $24 \mathrm{~h}$. The lyophilized NP powder with different lyoprotectants or concentrations $(2-10 \%)$ was resuspended in water to detect the ability to re-dissolve, and particle size and $\zeta$ potential were measured by DLS.

Cell lines and culture conditions. Sensitive K562 and HepG2 cells were cultured in RPMI-1640 medium supplemented with $10 \%(\mathrm{v} / \mathrm{v})$ FBS and $1 \%(\mathrm{v} / \mathrm{v})$ penicillin-streptomycin in a humidified atmosphere with $5 \% \mathrm{CO} 2$ at $37^{\circ} \mathrm{C}$. For the DOX-resistant K562 and HepG2 cell cultures, $1 \mu \mathrm{M}$ DOX was added in the RPMI-1640 medium every three passages to maintain drug resistance. The cells were used for experiments once they reached $80 \%$ confluency.

In vitro cytotoxicity assay. Cells were incubated at $37^{\circ} \mathrm{C}$ overnight in 96-well plates (Corning Inc., Corning, NY, USA) at a cell density of $5 \times 10^{3}$ cells/well. Medium was then removed and replaced with fresh medium containing blank NPs, free
Table I. Characteristics of the lipid-polymer hybrid nanoparticle at varying lipid/PLGA mass ratios.

\begin{tabular}{lccc}
\hline $\mathrm{W}_{\mathrm{PC}} / \mathrm{W}_{\text {PLGA }}, \%$ & Diameter, $\mathrm{nm}$ & $\mathrm{ZP}, \mathrm{mV}$ & PDI \\
\hline 10 & $77.62 \pm 1.24$ & $-28.8 \pm 1.95$ & $0.248 \pm 0.024$ \\
50 & $80.73 \pm 2.48$ & $-33.8 \pm 3.48$ & $0.221 \pm 0.019$ \\
100 & $77.02 \pm 2.96$ & $-35.4 \pm 5.52$ & $0.235 \pm 0.037$ \\
150 & $75.96 \pm 3.60$ & $-29.7 \pm 2.57$ & $0.184 \pm 0.017$ \\
200 & $98.37 \pm 2.56$ & $-20.4 \pm 4.23$ & $0.117 \pm 0.023$ \\
300 & $115.7 \pm 6.89$ & $-16.2 \pm 1.38$ & $0.132 \pm 0.041$ \\
400 & $147.7 \pm 4.88$ & $-17.3 \pm 1.96$ & $0.109 \pm 0.027$ \\
\hline
\end{tabular}

Data are presented as the mean \pm standard error of the mean $(n=3)$. PDI, polydispersity index; ZP, $\zeta$ potential; PC, phosphatidylcholine; PLGA, poly(D,L-lactide-co-glycolide).

DOX, free PSO, DOX + PSO or DOX + P-LPNs, and further incubated for $48 \mathrm{~h}$. PSO or the PSO-loaded NPs were at the equivalent PSO dose of 50, 25, 12.5, 6.25 and $3.12 \mu \mathrm{M}$, respectively. The amount of blank NPs was equivalent to the blank carrier in P-LPNs, which ranged from 6.25 to $50 \mu \mathrm{M}$. DOX used for sensitive K562 and HepG2 cells ranged from 0.006 to $12.5 \mu \mathrm{M}$, and for resistant $\mathrm{K} 562$ and HepG2 cells from 0.78 to $200 \mu \mathrm{M}$. A non-toxic concentration of $20 \mu \mathrm{M}$ PSO only was used in co-administration protocols with DOX in either the free form or in the NP formulations. Medium was removed at the end of the incubation period, and $20 \mu \mathrm{lCCK}-8$ solution was added to each well. Plates were further incubated for $3 \mathrm{~h}$. Then absorbance was measured at a wavelength of $420 \mathrm{~nm}$ using a 96-well plate reader (BioTek Instruments, Inc., Winooski, VT, USA). Cell toxicity was calculated according to the following equation: Cell viability $(\%)=\mathrm{Abs}_{\text {sample }} / \mathrm{Abs}_{\text {control }} \times 100$, where $\mathrm{Abs}_{\text {sample }}$ is the absorbance of cells in the presence of different formulations and $\mathrm{Abs}_{\text {control }}$ is the absorbance of cells in the absence of drug. The half maximal inhibitory concentration $\left(\mathrm{IC}_{50}\right)$ values were determined by fitting hyperbolic concentration response curves to data points.

Statistical analysis. Quantitative data are expressed as the mean \pm standard deviation. The statistical significance was determined by Student's t-test (between 2 groups) or by one-way analysis of variance ( $\geq 3$ groups) followed by Tukey's multiple comparison test, using GraphPad Prism 6.0 software (GraphPad Software, La Jolla, CA, USA). P $<0.05$ was considered to indicate a statistically significant difference.

\section{Results and Discussion}

Preparation and characterization of LPNs. PC and pegylated phospholipids (DSPE-PEG ${ }_{2000}$ ) were dissolved in ethanol to form the aqueous phase, which was heated at $37^{\circ} \mathrm{C}$ for dispersion. PLGA or PLGA/PSO was dissolved in acetonitrile to form the oil phase, which was then added to the preheated aqueous phase. The solution was subsequently stirred for $90 \mathrm{~min}$ to allow for the lipids to self-assemble and the organic solvent to evaporate. This allows a lipid monolayer to be formed around the PLGA/PSO core. The PLGA and PEG, as well as soybean 
A

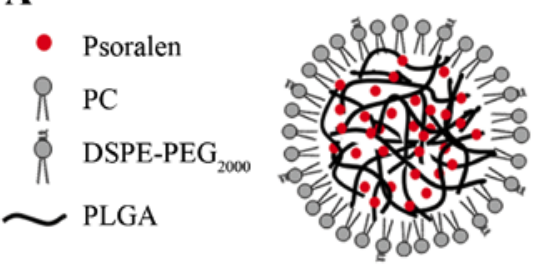

C

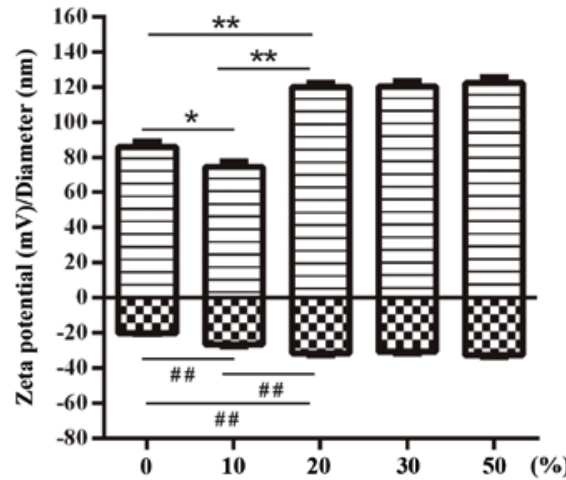

E

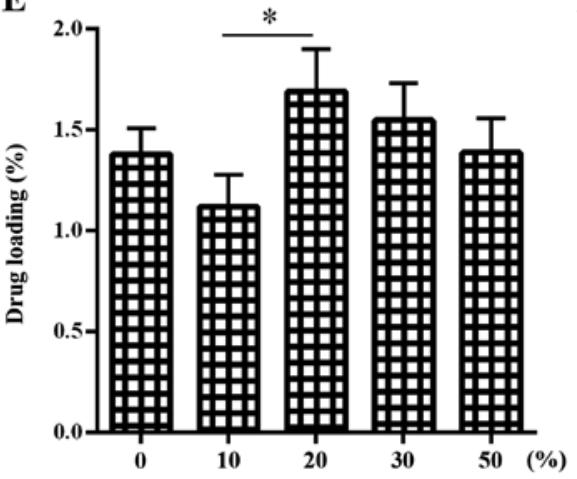

B

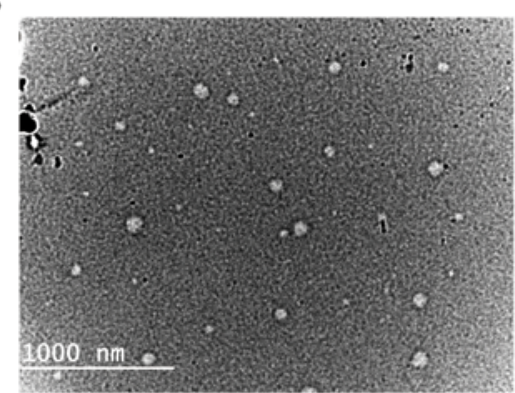

D

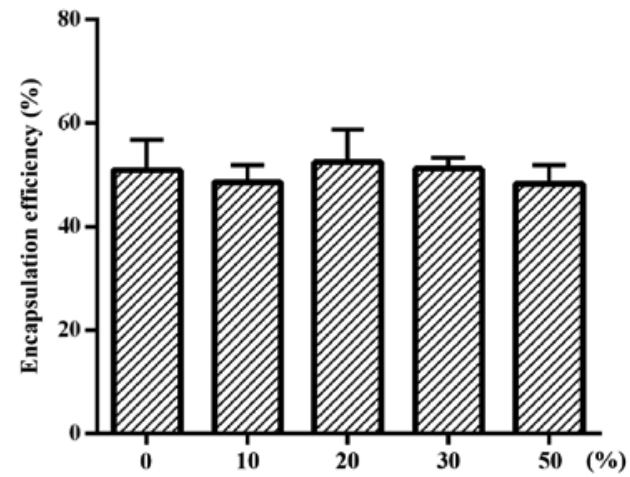

$\mathbf{F}$

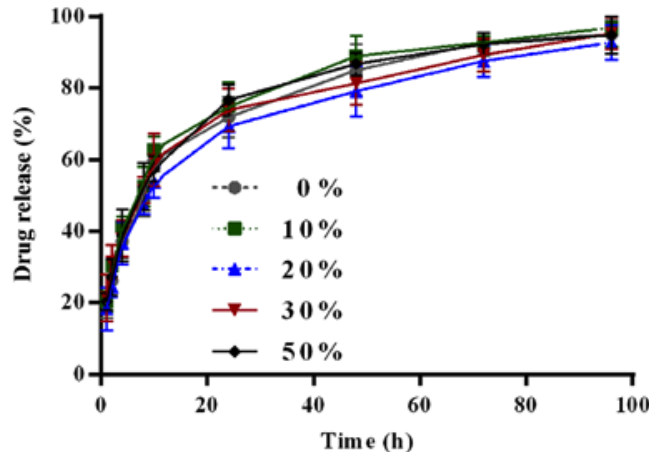

Figure 1. (A) Schematic illustration of the P-LPN structure featuring a lipid shell and a polymer core. (B) Transmission electron micrograph imaging of LPNs. Characteristics of the P-LPNs. (C) Changes in NP size and $\zeta$ potential, (D) effect on encapsulation efficiency, (E) influence on drug loading and (F) in vitro drug release profiles at various DSPE-PEG 2000 /lipid weight ratios. ${ }^{*} \mathrm{P}<0.05,{ }^{* *} \mathrm{P}<0.01$ and ${ }^{\# \#} \mathrm{P}<0.01$. NP, nanoparticle; PC, phosphatidylcholine; $\mathrm{DSPE}-\mathrm{PEG} 2000$, 1,2-distearoyl-sn-glycero-3-phosphoethanolamine-N-carboxy(polyethylene glycol)2000; PLGA, poly(D,L-lactide-co-glycolide); P-LPN, psoralen-loaded lipid-polymer hybrid nanoparticles.

PC, have been proven to be non-toxic and biocompatible by the FDA.

DLS was applied to characterize NP size, poly-dispersity and $\zeta$ potential in each preparation. The mean diameter of the NPs ranged from 60 to $80 \mathrm{~nm}$, while the $\zeta$ potential ranged between -20 and $-30 \mathrm{mV}$. PSO loading had an effect on the particle size and $\zeta$ potential. The schematic composition of a hybrid lipid-polymer NP and the TEM image of a preparation are shown in Fig. 1A and B, respectively. TEM images indicated that the NPs exhibited a well-defined spherical shape with mono-dispersion and no adhesion. Incorporation of PSO caused no significant morphological changes.

Determining the optimal lipid-polymer ratio in the preparation. The mean size, poly-dispersity and $\zeta$ potential of the LPNs at different lipid/PLGA ratios between 10 and $400 \%$ (w/w) are shown in Table I. Lipids, functioning as emulsifiers, stayed at the oil-water interface to lower the surface tension, and thus aid in producing the NP. At the weight (W)PC/WPLGA ratio of $10-200 \%$, the particle size ranged from 70 to $100 \mathrm{~nm}$, with polydispersity of $0.1-0.25$. However, NPs formed at $10 \%$ $\mathrm{W}_{\mathrm{PC}} / \mathrm{W}_{\mathrm{PLGA}}$ were observed to sediment to the bottom of the bottle overnight (data not shown), likely due to coalescence and precipitation of the polymer core resulting from incomplete lipid coverage $(8,13)$. Increasing the $\mathrm{W}_{\mathrm{PC}} / \mathrm{W}_{\mathrm{PLGA}}$ ratio above 2 significantly increased the size, while the $\zeta$ potential decreased. An increasing lipid-PLGA ratio thus influenced size and $\zeta$ potential in an inverse manner.

An appropriate amount of lipids shielded the hydrophobic PLGA polymer core to increase stability. However, an excess of lipids thickened the lipid layer, thereby increasing the particle size and reducing the $\zeta$ potential (8). Thus, in terms of 
A

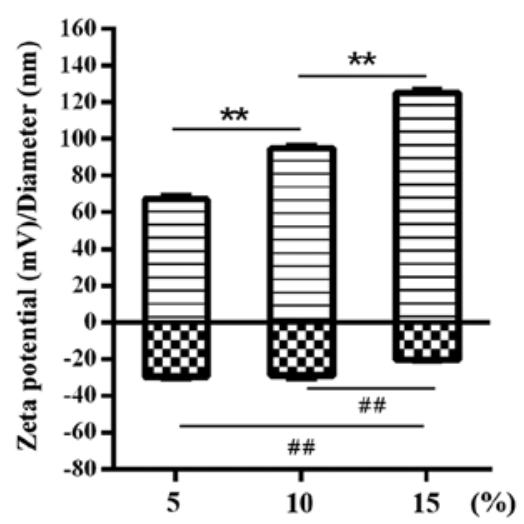

B

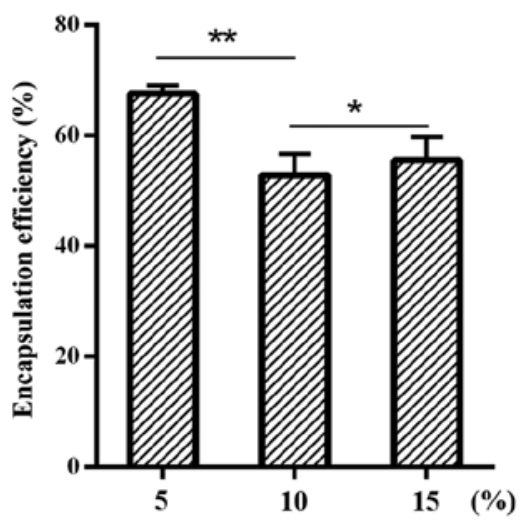

C

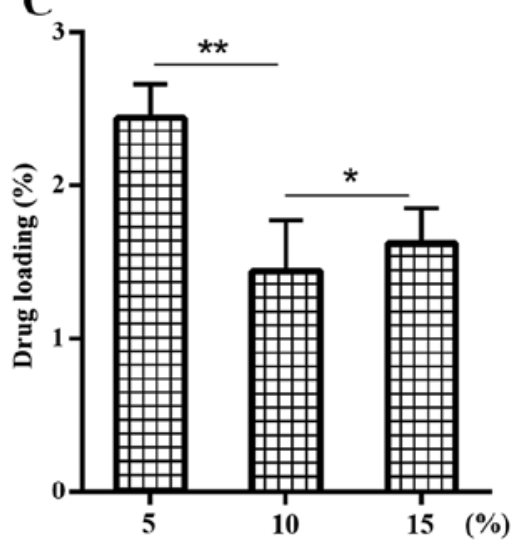

D

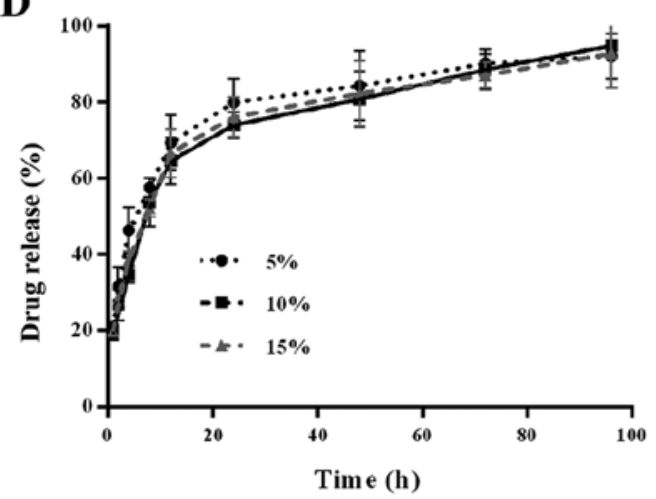

E

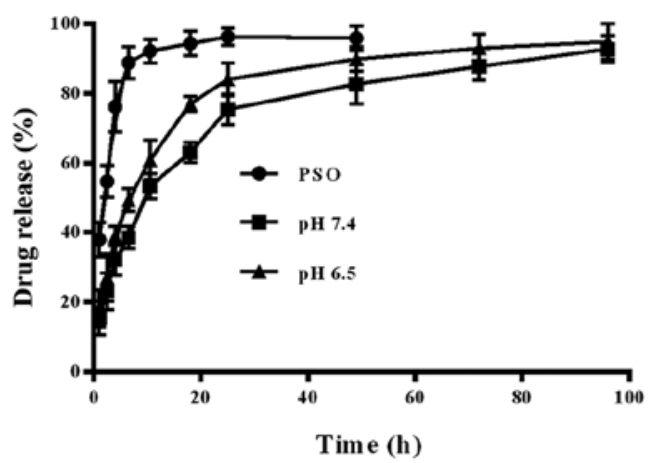

Figure 2. Characteristics of P-LPNs. (A) Influence of PSO on nanoparticle size and $\zeta$ potential, (B) effect on encapsulation efficiency and (C) drug loading, (D) in vitro drug release profile at various $\mathrm{PSO}$ concentrations. (E) In vitro drug release profiles of free $\mathrm{PSO}$ and $\mathrm{P}-\mathrm{LPNs}$ at different $\mathrm{pH}$. $\mathrm{P}<0.05$, ${ }^{* *} \mathrm{P}<0.01$. ${ }^{\# \#} \mathrm{P}<0.01$. P-LPN, psoralen-loaded lipid-polymer hybrid nanoparticles; PSO, psoralen.

NP size and $\zeta$ potential, $100 \% \mathrm{~W}_{\mathrm{PC}} / \mathrm{W}_{\mathrm{PLGA}}$ was determined as optimal and chosen for subsequent experiments.

Influence of the lipid-PEG/lipid ratio on NP properties. Next, the effect of the lipid-PEG/lipid (DSPE-PEG ${ }_{2000} / \mathrm{PC}$ ) mass ratio on NP characteristics was investigated, with the PLGA/ lipid weight ratio constant kept at $100 \%$. Data in Fig. 1C-E show the NP size, EE and DL at different lipid-PEG/lipid mass ratios. The amount of total lipids was kept constant, while the ratio between the two components was changed from only PC to a $1+1$ ratio of PC and DSPE-PEG ${ }_{2000}$. NP size decreased at $10 \%$ DSPE-PEG $_{2000}$. This decrease was statistically significant with a P-value of $<0.05$. At 20,30 and $50 \%$ the NP size increased $(\mathrm{P}<0.01)$. Values at 20,30 and $50 \%$ did not exhibit a significant difference. By contrast, a decrease in $\zeta$ potential was observed in the samples containing 10 and $20 \%$ DSPE$-\mathrm{PEG}_{2000}$, but no further decrease was observed at 30 and $50 \%$. Drug load varied with NP size, whereby statistical significance was only reached for the $20 \%$ data point. While DL and NP size changed, the EE was close in all samples.

The in vitro drug release profiles of the P-LPNs with different lipid-PEG/lipid mass ratios are presented in Fig. 1F. When the lipid-PEG/lipid mass ratio was varied from 0 to $50 \%(\mathrm{w} / \mathrm{w})$, only small differences in the in vitro drug release rates were observed, which demonstrated that different amounts of PEGylated lipid in the lipid-polymer NPs did not significantly affect PSO release. The half-lives for PSO release were $11.2,9.5,11.9,10.4$ and $10.0 \mathrm{~h}$, respectively, when the $\mathrm{DSPE}_{-} \mathrm{PEG}_{2000} / \mathrm{PC}$ ratio was increased from 0 to $50 \%$. Thus, the ratio of DSPE-PEG ${ }_{2000} / \mathrm{PC}$ had a minor effect on the drug release properties of the NPs. At $120 \mathrm{~h},>90 \%$ of the PSO had been released in a sustained manner. Considering the EE and DL, an intermediate lipid-PEG/lipid ratio of $1+4$ was chosen for further characterization of the formulations.

Influence of initial drug amount on NP properties. Another parameter, which may potentially affect NP size, is the PSO amount used in the preparation process. At a fixed $100 \%$ lipid/ polymer mass ratio and a 20\% lipid-PEG/lipid mass ratio, 5, 10 and $15 \% \mathrm{PSO} /($ lipid + PLGA) were encapsulated.

Fig. 2A shows that the NPs increased in size when the amount of PSO was increased. The $\zeta$ potential decreased at $15 \%$ PSO $(\mathrm{P}<0.01)$. An increase in PSO did not change the EE and DL. A significant increase in EE and DL was observed in the samples containing 5\% PSO as compared with those containing 10 or $15 \%$ (Fig. 2B and C). Drug release was also comparable, but no significant differences in the in vitro drug release rates of different samples were observed (Fig. 2D). The results indicated that, in the present study, the volume of the core polymer and the surface area of the NPs determined the DL, which is in agreement with previous reports from the literature $(21,22)$. Hence, the mass ratio of PSO to lipid/PLGA of $5 \%$ was chosen for subsequent experiments. 
$\mathbf{A}$

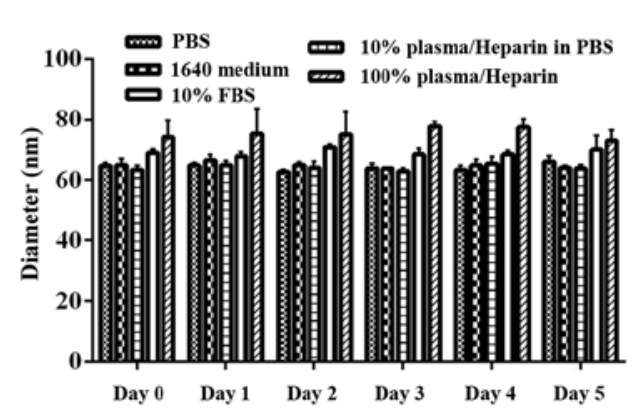

B

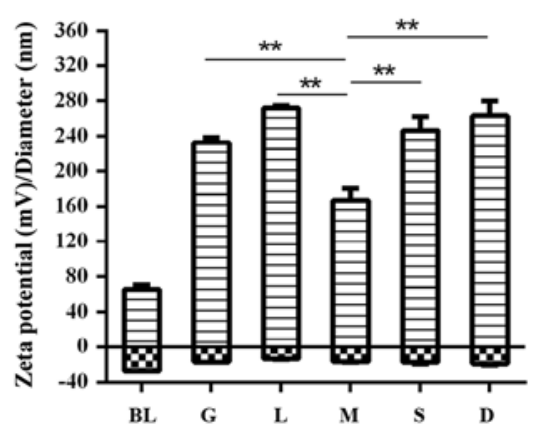

C

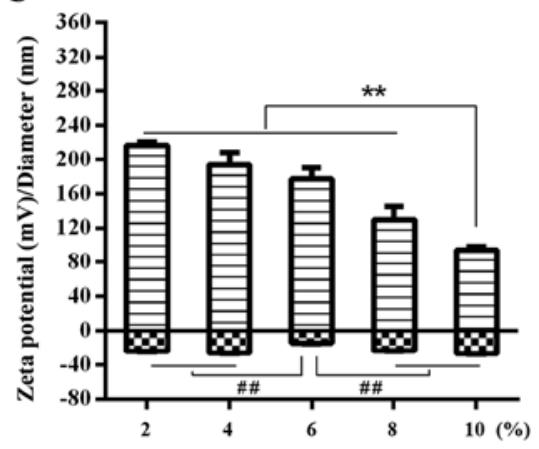

Figure 3. Post-formulation stability of P-LPNs. (A) In vitro stability of P-LPNs over 5 days. NPs were incubated in PBS or RPMI-1640 medium. 10\% (v/v) fetal bovine serum, $10 \%(\mathrm{v} / \mathrm{v})$ human heparin plasma in PBS and $100 \%$ human heparin plasma were also used. Incubation proceeded for up to 5 days at $37^{\circ} \mathrm{C}$. An aliquot of the NP suspension was used to measure NP size using Malvern dynamic light scattering. (B) Post-formulation stability of the P-LPNs following freeze-drying and lyophilization with five cryroprotectants $(5 \%, \mathrm{w} / \mathrm{v})$, glucose, lactose, mannitol, sucrose and D-trehalose. (C) Post-formulation stability of the P-LPNs assessed following lyophilization in mannitol at concentrations of $2,4,6,8$ and $10 \%(\mathrm{w} / \mathrm{v}) .^{* *} \mathrm{P}<0.01$ indicates statistically significant differences between $5 \%$ mannitol and the other lyoprotectants, including glucose, lactose, mannitol, sucrose and D-trehalose. ${ }^{\# \#} \mathrm{P}<0.01$ indicates a statistically significant difference in the $\zeta$ potential. BL, before lyophilization; G, glucose; L, lactose; M, mannitol; S, sucrose; D, D-trehalose; PBS, phosphate-buffered saline; FBS, fetal bovine serum; NP, nanoparticle; P-LPN, psoralen-loaded lipid-polymer hybrid nanoparticles.

Table II. $\mathrm{IC}_{50}$ value for free DOX alone or in combination with free PSO or LPNs in K562 and HepG2 cells.

\begin{tabular}{lccrr}
\hline & \multicolumn{3}{c}{$\mathrm{IC}_{50}, \mathrm{nmol} / \mathrm{l}^{\mathrm{a}}$} \\
\cline { 2 - 5 } Sample & K562/S & K562/DOX & HepG2/S & HepG2/DOX \\
\hline DOX in solution & $19.8 \pm 0.31$ & $41.200 \pm 0.26$ & $229.8 \pm 0.57$ & $74.930 \pm 0.82$ \\
DOX + PSO & $12.2 \pm 0.43$ & $6.190 \pm 0.57$ & $245.1 \pm 0.73$ & $6.777 \pm 0.46$ \\
DOX + P-LPNs & $/$ & $2.860 \pm 0.12$ & $/$ & $3.254 \pm 0.69$ \\
Resistance index $^{\mathrm{b}}$ & & 2086 & 326
\end{tabular}

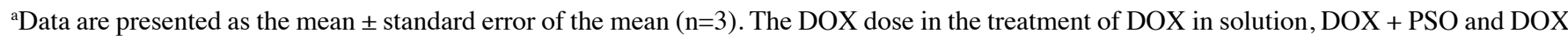
+ P-LPNs was equivalent. ${ }^{b}$ Resistance index $=\mathrm{IC}_{50}$ of DOX-resistant cells/ $/ \mathrm{IC}_{50}$ of sensitive cells. $\mathrm{IC}_{50}$, half maximal inhibitory concentration; DOX, doxorubicin; PSO, psoralen; P-LPNs, PSO-loaded lipid-polymer hybrid nanoparticle; S, sensitive.

In vitro drug release profile. In vitro release of PSO from P-LPNs was monitored over $96 \mathrm{~h}$ using the dialysis-bag method. P-LPNs were thereby compared to free PSO in the dialysis bag. A number of studies have shown that tumor tissue represents an acidic microenvironment due to insufficient oxygen supply and thus anaerobic energy provision. Generally the $\mathrm{pH}$ in tumors lies between 6.0 and 6.9, while in non-neoplastic tissue it is maintained at a $\mathrm{pH}$ value of between 7.35 and 7.45 (23,24). Thus, PBS at pH 7.4 and 6.5 was used as the dialysis medium to study the sustained release from NPs at these different $\mathrm{pH}$ conditions. As shown in Fig. 2E, free PSO in solution was basically removed from the dialysis bag within the first $6 \mathrm{~h}$, while the P-LPNs showed delayed release characteristics. The release characteristics were improved due to the nano-formulation, and the cumulative release of PSO exceeded $90 \%$ after $96 \mathrm{~h}$.

The half-lives of PSO release in P-LPNs were 11.9 and $7.5 \mathrm{~h}$ at $\mathrm{pH} 7.4$ and 6.5, respectively (Fig. 2E). In the first $10 \mathrm{~h}$, cumulative release reached $\sim 53 \%$ at $\mathrm{pH} 7.4$ and $61 \%$ at $\mathrm{pH} 6.5$ for the P-LPNs. Drug release at pH 6.5 was thus faster than at $\mathrm{pH} 7.4$, probably due to a more rapid degradation of surface lipids in a more acidic environment. These findings are in accordance with data showing that internalization of drugloaded nanocarriers into cells is stimulated by degradation in the acidic environment of lysosomes (25).

In vitro stability of LPNs. In order to investigate the longterm stability and protein binding of LPNs, a formulation of $100 \%$ lipid/PLGA mass ratio, 20\% lipid-PEG/lipid mass ratio and $5 \% \mathrm{PSO} /$ (lipid + PLGA) mass ratio were chosen. Lipidpolymer hybrid NPs were incubated in PBS, RPMI-1640 medium, RPMI-1640 medium containing 10\% FBS, $10 \%$ (v/v) human plasma in PBS or $100 \%$ human plasma at $37^{\circ} \mathrm{C}$ for $120 \mathrm{~h}$. Fig. 3A shows that size and polydispersity of NPs incubated in either PBS or RPMI-1640 medium did not change significantly. This indicates that a monolayer comprised of PEGylated lipid molecules stabilizes the polymer core and prevents NP aggregation over the course of $120 \mathrm{~h}$.

In $10 \%$ FBS and in 10 or $100 \%$ human plasma, the change in NP size was used as a criterion for evaluation of protein adsorption and bio-fouling. As shown in Fig. 3A, the hybrid NPs were stable in $10 \%$ FBS, as they retained a size of $64 \pm 4 \mathrm{~nm}$ (mean \pm SEM polydispersity $=0.22 \pm 0.11 ; \mathrm{n}=3$ ), over $120 \mathrm{~h}$. A small increase in size was observed in $100 \%$ 
A

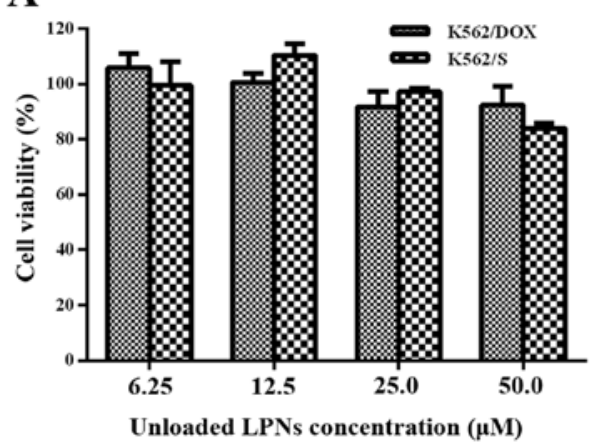

C

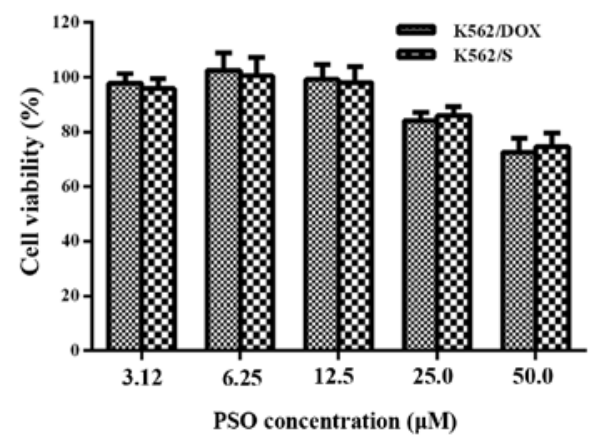

E

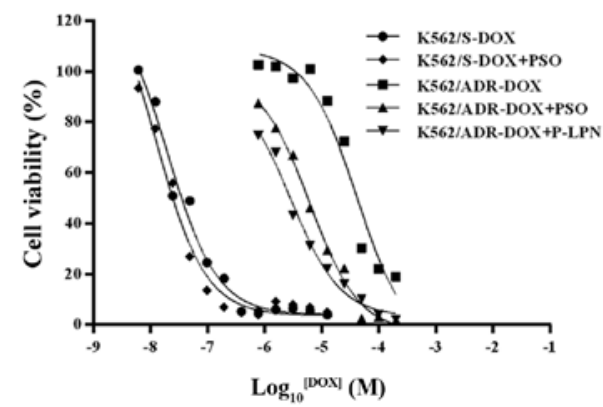

B

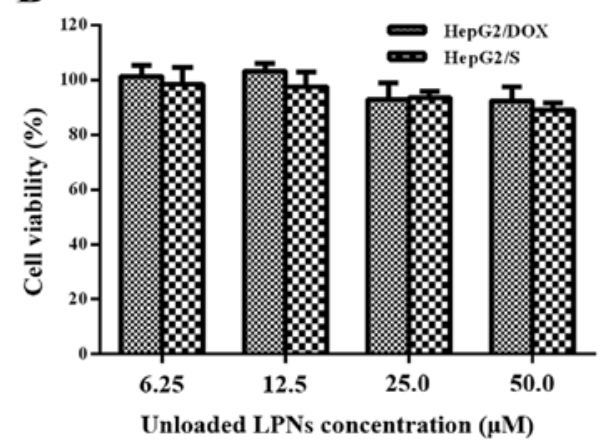

D

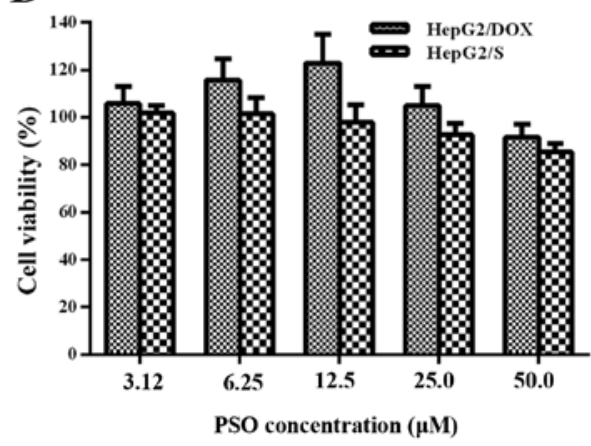

F

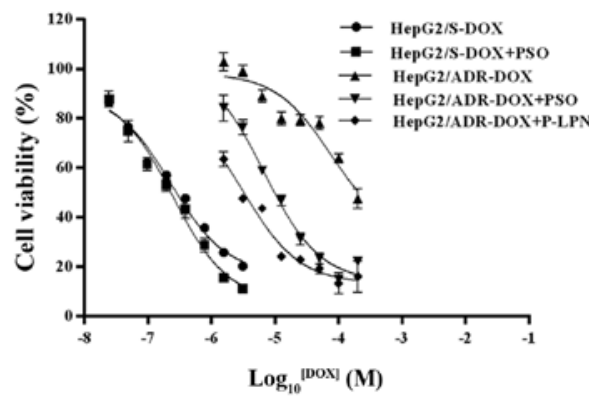

Figure 4. In vitro cell viability of unloaded lipid-polymer hybrid nanoparticles on sensitive and DOX-resistant (A) K562 and (B) HepG2 cells at a PSO equivalent concentration ranging from 6.25 to $50 \mu \mathrm{M}$, respectively. Cytotoxicity of the modulator PSO on sensitive and DOX-resistant (C) K562 and (D) HepG2 cells at concentrations between 3.12 and $50 \mu \mathrm{M}$. Cytotoxicity of DOX + PSO, DOX + P-LPNs on DOX-resistant (E) K562 and (F) HepG2 cells in comparison with free DOX. Cell viability subsequent to $48 \mathrm{~h}$ was measured using the Cell Counting Kit- 8 assay. Survival percentage was compared with that of untreated controls and expressed as a percentage of these controls. Data points represent the mean \pm standard error of the mean. Wherever error bars are not visible, they lie within the symbols. DOX, doxorubicin; PSO, psoralen; P-LPN, PSO-loaded lipid-polymer hybrid nanoparticles; S, sensitive.

human serum as compared with that in $10 \%$ human serum, but without statistical significance. These results suggested that PEGylation of the NP prevents protein adsorption on the NP surface. The formulation has appropriate stability, which was consistent with the results of a previous study (20). In addition to improving the solubility and stability of the nanocarriers in aqueous solution, PEGylation has been reported to minimize opsonisation during circulation in the blood stream and the reticuloendothelial systems in the liver, spleen, and kidneys, thus increasing NP circulation time $(26,27)$.

Optimization of the lyoprotectant formulation for LPNs. Five different lyoprotectants (5\%, w/v), glucose, lactose, mannitol, sucrose and D-trehalose, were used during the lyophilisation procedure to identify the one that was superior. Fig. 3B shows that particle size increased by more than two times and $\zeta$ potential sharply increased in all samples due to the addition of lyoprotectant. Mannitol resulted in the lowest size following re-dissolution $(167 \pm 13.2 \mathrm{~nm})$ and was therefore chosen as the optimal lyoprotectant for further experiments. When the concentration of mannitol was increased from $2 \%$ to $10 \%(\mathrm{w} / \mathrm{v})$, a more than two-fold decrease in particle size was observed (down to $100 \mathrm{~nm}$ ). A concentration of $10 \%$ mannitol resulted in the smallest size following reconstitution $(94.4 \pm 4.1 \mathrm{~nm})$, which thus most closely matched the original particle size (Fig. 3C). The $\zeta$ potential showed no significant difference at concentrations of 2, 4, 8 and $10 \%$, but was lower at $6 \%(\mathrm{P}<0.01)$.

In vitro cytotoxicity of LPNs. In vitro cytotoxicity of drug-free LPNs, free PSO and P-LPN was assessed in the K562 and the HepG 2 cell lines over 48 h, using the CCK- 8 method. The 
unloaded LPNs at concentrations between 6.25 and $50 \mu \mathrm{M}$ (Fig. 4A and B) and the modulator PSO alone at concentrations between 3.12 and $25 \mu \mathrm{M}$ (Fig. 4C and D) did not exhibit any cytotoxic effect. The DOX IC50 was $19.8 \mathrm{nM}$ in the drug-sensitive K562 cells, $41.2 \mu \mathrm{M}$ in the DOX-resistant K562 cells, $230 \mathrm{nM}$ in the sensitive HepG2 cells and $74.9 \mu \mathrm{M}$ in the DOX-resistant HepG2 cells (Table II). The resistance indices of DOX-resistant K562 and HepG2 cells were 2,086 and 326, respectively, which demonstrated that the resistant cells were highly resistant to DOX. Co-administration of DOX and P-LPN at an identical concentration of $20 \mu \mathrm{M}$ PSO exhibited significantly higher cytotoxicity compared with that of free DOX or (DOX + PSO) at all drug concentrations (Fig. 4E and F). This demonstrated that co-administration of DOX and P-LPNs was superior to the same regimen in solution in all cases. The NP formulation showed a 14- and 23-fold stronger effect in inhibiting cell growth in resistant K562 and HepG2 cells compared with free DOX (19.8 and $230 \mathrm{nM}$, respectively), and a 2.2- and 2.1-fold stronger effect than free DOX plus PSO (6.2 and $6.8 \mu \mathrm{M})$. These changes were statistically significant $(\mathrm{P}<0.05)$.

In the present study, a formulation of lipid-polymer hybrid nanocarriers with a lipid shell and a PLGA core for sustained and controlled release of PSO was developed. Parameters that could have an effect on the preparation of NPs were investigated to optimize the formulation. Next, the physical properties and cytotoxicity were studied. P-LPNs were prepared using the ESE method. It was found that the amount of lipid served an important role in particle size and stability. A WPC/WPLGA of 1 proved optimal. PEGylated surface lipids had a minor effect on in vitro drug release, while high PEG surface density increased particle size and $\zeta$ potential. The optimal lipidPEG/lipid mass ratio was determined to be $20 \%$. When the $\mathrm{PSO} /$ (lipid + PLGA) mass ratio was increased, size increased, and $\zeta$ potential decreased at $15 \%$ PSO. However, there were no apparent changes in EE and DL. Optimized NP formulations were evaluated for physical stability in five different media in the absence and presence of human plasma. There was no marked change in NP size following a 5-day incubation period, which suggested that the formulation had sufficient stability in PBS, medium and plasma. Mannitol at a concentration of $10 \%$ $(w / v)$ was found to be the best lyoprotectant. Unloaded LPNs were well tolerated by the human model cell lines in vitro. Compared with free PSO, P-LPNs showed enhanced toxicity of the chemotherapeutic drug DOX in DOX-resistant cells. In summary, LPNs hold promise as a drug delivery system for effective chemotherapy. Further studies on the in vivo efficacy of these formulations will be required prior to broader use in a clinical context.

\section{Acknowledgements}

Not applicable.

\section{Funding}

This study was supported by the National Natural Science Foundation of China (grant no. 81273707), the Ministry of Education in the New Century Excellent Talents (grant no. NECT-12-0677), the Natural Science
Foundation of Guangdong (grant nos. S2013010012880 and 2016A030311037), the Science and Technology Program of Guangzhou (grant nos. 2014J4500005 and 201704030141), the Science Program of the Department of Education of Guangdong (grant nos. 2013KJCX0021 and 2015KGJHZ012), the Science and Technology Program of Guangdong (grant no. 2015A050502027), and the Special Project of International Scientific and Technological Cooperation in Guangzhou Development District (grant no. 2017GH16).

\section{Availability of data and materials}

All data generated or analyzed during this study are included in this published article.

\section{Authors' contributions}

SPCC and YC designed the research and took responsible for all aspects of the study. YY and TC performed the majority of the research work and wrote the initial draft of the paper. LB assisted in some of the experiments. PC analyzed the majority of the data and revised the manuscript critically for important intellectual content. RC contributed to refining the ideas, performing additional analyses and finalizing the paper. All authors read and approved the final manuscript.

\section{Ethics approval and consent to participate}

Not applicable.

\section{Patient consent for publication}

Not applicable.

\section{Competing interests}

The authors declare that they have no competing interests.

\section{References}

1. Rogueda PG and Traini D: The nanoscale in pulmonary delivery. Part 1: Deposition, fate, toxicology and effects. Expert Opin Drug Deliv 4: 595-606, 2007.

2. Cooper DL, Conder CM and Harirforoosh S: Nanoparticles in drug delivery: Mechanism of action, formulation and clinical application towards reduction in drug-associated nephrotoxicity. Expert Opin Drug Deliv 11: 1661-1680, 2014.

3. Yuan Y, Cai T, Xia X, Zhang R, Chiba P and Cai Y: Nanoparticle delivery of anticancer drugs overcomes multidrug resistance in breast cancer. Drug Deliv 23: 3350-3357, 2016.

4. Jia F, Liu X, Li L, Mallapragada S, Narasimhan B and Wang Q: Multifunctional nanoparticles for targeted delivery of immune activating and cancer therapeutic agents. J Control Release 172: 1020-1034, 2013.

5. Liu Y, Pan J and Feng SS: Nanoparticles of lipid monolayer shell and biodegradable polymer core for controlled release of paclitaxel: Effects of surfactants on particles size, characteristics and in vitro performance. Int J Pharm 395: 243-250, 2010.

6. Mandal B, Bhattacharjee H, Mittal N, Sah H, Balabathula P, Thoma LA and Wood GC: Core-shell-type lipid-polymer hybrid nanoparticles as a drug delivery platform. Nanomedicine (Lond) 9: 474-491, 2013

7. Vyas S, Rai S, Paliwal R, Gupta PN, Khatri K, Goyal AK and Vaidya B: Solid lipid nanoparticles (SLNs) as a rising tool in drug delivery science: One step up in nanotechnology. Curr Nanosci 4: $30-44,2008$ 
8. Cheow WS and Hadinoto K: Factors affecting drug encapsulation and stability of lipid-polymer hybrid nanoparticles. Colloids Surf B Biointerfaces 85: 214-220, 2011.

9. Mukherjee B, Satapathy BS, Mondal L, Dey NS and Maji R: Potentials and challenges of active targeting at the tumor cells by engineered polymeric nanoparticles. Curr Pharm Biotechnol 14: 1250-1263, 2013.

10. Thevenot J, Troutier AL, David L, Delair T and Ladavière C: Steric stabilization of lipid/polymer particle assemblies by poly(ethylene glycol)-lipids. Biomacromolecules 8: 3651-3660, 2007.

11. Zhang L, Zhu D, Dong X, Sun H, Song C, Wang C and Kong D: Folate-modified lipid-polymer hybrid nanoparticles for targeted paclitaxel delivery. Int J Nanomedicine 10: 2101-2114, 2015.

12. Feng S-S and Chien S: Chemotherapeutic engineering: Application and further development of chemical engineering principles for chemotherapy of cancer and other diseases. Chem Eng Sci 58: 4087-4114, 2003.

13. Chan JM, Zhang L, Yuet KP, Liao G, Rhee JW, Langer R and Farokhzad OC: PLGA-lecithin-PEG core-shell nanoparticles for controlled drug delivery. Biomaterials 30: 1627-1634, 2009.

14. Hsieh MJ, Chen MK, Yu YY, Sheu GT and Chiou HL: Psoralen reverses docetaxel-induced multidrug resistance in A549/D16 human lung cancer cells lines. Phytomedicine 21: 970-977, 2014.

15. Jiang J, Wang X, Cheng K, Zhao W, Hua Y, Xu C and Yang Z: Psoralen reverses the $\mathrm{P}$-glycoprotein-mediated multidrug resistance in human breast cancer MCF-7/ADR cells. Mol Med Rep 13: 4745-4750, 2016.

16. Cai J, Chen S, Zhang W, Hu S, Lu J, Xing J and Dong Y: Paeonol reverses paclitaxel resistance in human breast cancer cells by regulating the expression of transgelin 2. Phytomedicine 21 : 984-991, 2014.

17. Jambhrunkar S, Qu Z, Popat A, Karmakar S, Xu C and Yu C: Modulating in vitro release and solubility of griseofulvin using functionalized mesoporous silica nanoparticles. J Colloid Interface Sci 434: 218-225, 2014.
18. Jeong H, Samdani KJ, Yoo DH, Lee DW, Kim NH, Yoo IS and Lee JH: Resveratrol cross-linked chitosan loaded with phospholipid for controlled release and antioxidant activity. Int J Biol Macromol 93: 757-766, 2016.

19. Tahir N, Madni A, Balasubramanian V, Rehman M, Correia A, Kashif PM, Mäkilä E, Salonen J and Santos HA: Development and optimization of methotrexate-loaded lipid-polymer hybrid nanoparticles for controlled drug delivery applications. Int $\mathbf{J}$ Pharm 533: 156-168, 2017.

20. Zhang L, Chan JM, Gu FX, Rhee JW, Wang AZ, RadovicMoreno AF, Alexis F, Langer $\mathrm{R}$ and Farokhzad OC: Self-assembled lipid - polymer hybrid nanoparticles: A robust drug delivery platform. ACS Nano 2: 1696-1702, 2008.

21. Mu L and Feng SS: Vitamin E TPGS used as emulsifier in the solvent evaporation/extraction technique for fabrication of polymeric nanospheres for controlled release of paclitaxel (Taxol). J Control Release 80: 129-144, 2002.

22. Ruan G and Feng SS: Preparation and characterizations of PLA-PEG-PLA micro-spheres for controlled release of paclitaxel. Biomaterials 24: 5037-5044, 2003.

23. Abaza M and Luqmani YA: The influence of $\mathrm{pH}$ and hypoxia on tumor metastasis. Expert Rev Anticancer Ther 13: 1229-1242, 2013.

24. Schornack PA and Gillies RJ: Contributions of cell metabolism and $\mathrm{H}^{+}$diffusion to the acidic $\mathrm{pH}$ of tumors. Neoplasia 5: 135-145, 2003.

25. Saraswathy M and Gong S: Different strategies to overcome multidrug resistance in cancer. Biotechnol Adv 31: 1397-1407, 2013.

26. Åslund AKO, Sulheim E, Snipstad S, von Haartman E, Baghirov H, Starr N, Kvåle Løvmo M, Lelú S, Scurr D, Davies CL, et al: Quantification and qualitative effects of different PEGylations on Poly(butyl cyanoacrylate) Nanoparticles. Mol Pharm 14: 2560-2569, 2017.

27. Harris JM and Chess RB: Effect of pegylation on pharmaceuticals. Nat Rev Drug Discov 2: 214-221, 2003. 